\title{
INTERFAZ WEB USABLE: HERRAMIENTA TECNOLÓGICA PARA EL PROCESO DE ENSEÑANZA-APRENDIZAJE
}

Ricardo Adán Salas Rueda': Universidad La Salle, México. adansalas@hotmail.com

\section{RESUMEN:}

Los avances tecnológicos están propiciando cambios significativos en la forma de planear y organizar las actividades en las instituciones educativas. Los docentes se están convirtiendo en el elemento clave durante la transformación de los entornos virtuales de enseñanza-aprendizaje. Actualmente, los estudiantes que cursan la asignatura de Algorítmica y principios de programación en una universidad ubicada al sur del Distrito Federal, México presentan dificultades para entender los conceptos relacionados con las Estructuras de control y el Lenguaje de programación C. Por esta razón, el docente encargado de esta materia decidió mejorar la Interfaz Web Usable (IWU) por medio de la accesibilidad dando origen al IWU 2.0 cuya finalidad es brindar a los usuarios diversos contenidos audiovisuales en los idiomas español e inglés, los cuales se adecuan a sus características y necesidades. El diseño de la IWU 2.0 emplea los principios de la accesibilidad denominados Web Contents Accessibility Guidelines (WCAG 2) relacionados con el uso de la información perceptible, operable, comprensible y robusta. Esta investigación se apoya en el Enfoque cuantitativo por medio de la Prueba $T$ con el nivel de significancia de 0.1 y 0.05 para analizar el comportamiento de 18 estudiantes a través de la aplicación de 2 cuestionarios relacionados con las Estructuras de control: preprueba (sin uso de la tecnología) y postprueba donde se utiliza la IWU 2.0 como apoyo didáctico durante el curso presencial.

PALABRAS CLAVE: Educación Superior - Tecnología de la información - Interfaz Web - Accesibilidad de los contenidos audiovisuales

\footnotetext{
${ }^{1}$ Ricardo Adán Salas Rueda: Doctor en Diseño de Nuevas Tecnologías, egresado de la Universidad Autónoma Metropolitana (UAM) en el año 2014. Durante 13 años ha impartido diversos cursos relacionados con la tecnología y las matemáticas en el nivel de licenciatura y posgrado.

Correo: adansalas@hotmail.com
} 


\title{
USABLE WEB INTERFACE: TECHNOLOGICAL TOOL FOR TEACHING-LEARNING
}

\begin{abstract}
:
Technological advances are leading to significant changes in the way of planning and organizing activities in educational institutions. Teachers are becoming the key element in the transformation of virtual environments for teaching and learning. Currently, students taking the subject of Algorithms and programming principles in a university located south of Mexico City, Mexico have difficulty understanding the concepts related control structures and programming language $\mathrm{C}$. For this reason, the teacher charge of this matter decided improve Usable Web Interface (IWU) via accessibility giving rise to IWU 2.0 aimed at providing users with diverse audiovisual content in Spanish and English languages, which are appropriate to their characteristics and needs. The design of the IWU 2.0 employs the principles of accessibility called Web Contents Accessibility Guidelines (WCAG 2) related to the use of perceptible, operable, understandable and robust information. This research is based on the quantitative approach through $T$ test with significance level of 0.1 and 0.05 to analyze the behavior of 18 students through the application of two questionnaires related to Control Structures: pretest (unused technology) and posttest where IWU 2.0 is used as a teaching support during the classroom course.
\end{abstract}

KEY WORDS: Higher Education - Information Technology - Web Interface Accessibility of audiovisual content.

\section{INTRODUCCIÓN}

En México, diversas universidades están enfocadas en la formación académica con base en el desarrollo de las competencias y están empezando a considerar en la planeación estratégica al segmento compuesto por personas adultas que combinan las actividades educativas con las laborables, lo cual ha originado la creación de las licenciaturas ejecutivas. En este nuevo modelo de enseñanza-aprendizaje, la gran mayoría de los alumnos han dejado de estudiar por más de ocho años lo cual ha provocado que existan obstáculos académicos durante el reingreso al contexto educativo. Por esta razón, las instituciones de Educación Superior han decidido capacitar a los docentes por medio de diversos cursos vinculados con el desarrollo de las competencias, la utilización de la tecnología vanguardia y el uso del diseño instruccional.

En particular, el profesor encargado de la asignatura "Algorítmica y principios de programación" diseño la Interfaz Web Usable (IWU) en el año 2013 con el propósito de facilitar el proceso de educativo relacionado con las Estructuras de control. Con el paso del tiempo, el docente se ha dado cuenta que la IWU versión 1.0 necesita ser actualizada en los aspectos relacionados con el diseño de la interfaz web y los 
contenidos audiovisuales considerando los criterios de la accesibilidad. Es importante mencionar que la IWU versión 2.0 cuenta con el registro público del derecho de autor No: 03-2014-112809320900-01. La Figura 1 presenta la versión 1.0 y 2.0 de la IWU.

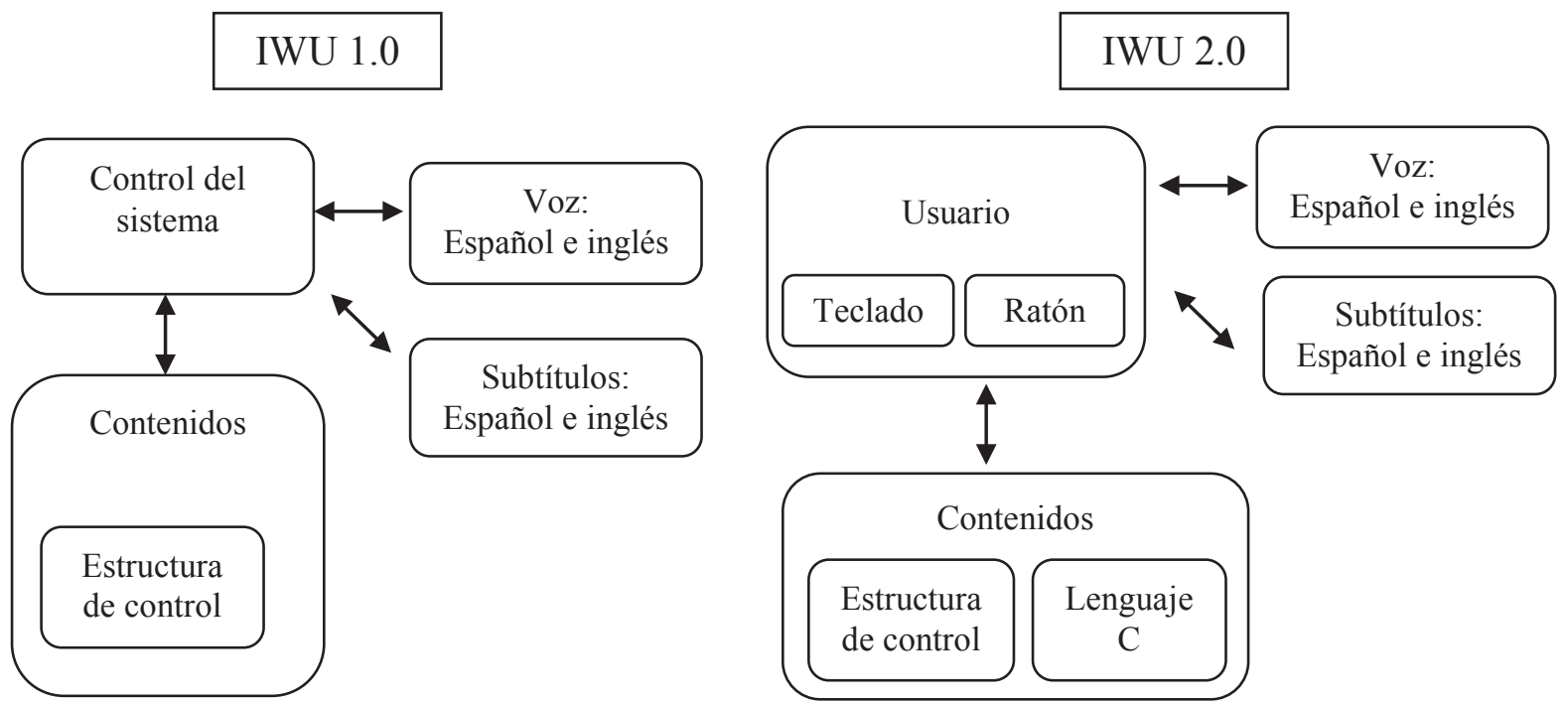

Figura 1: IWU versión 1.0 y 2.0.

Fuente: Diseño propio 2014

Como se puede observar en la Figura 1, la IWU 2.0 permite al usuario configurar los contenidos audiovisuales vinculados con las Estructuras de control y el Lenguaje de programación C de acuerdo a sus necesidades (accesibilidad).

De acuerdo con Shirogane, Mori , Iwata y Fukazawa (2008), la accesibilidad se refiere a que las personas pueden usar la tecnología de la información para cubrir sus necesidades por medio de las recomendaciones Web Contents Accessibility Guidelines(WCAG). Además estos autores destacan la importancia de utilizar el teclado, la estética (el uso del color y las propiedades de la fuente) y las alternativas textuales para facilitar la navegación y transmisión de la información en la web.

En resumen, el docente encargado de la asignatura "Algorítmica y principios de programación" desea analizar el comportamiento que presentan los estudiantes (conocimiento) sobre las Estructuras de Control y el Lenguaje de programación C al utilizar la IWU 2.0.

\section{OBJETIVOS}

Esta investigación relacionada con el uso de la tecnología de vanguardia (IWU 2.0) en el proceso de enseñanza-aprendizaje presenta como objetivos:

1. Diseñar la IWU 2.0 considerando el aspecto de la accesibilidad de los contenidos audiovisuales

2. Construir los contenidos audiovisuales sobre las Estructuras de Control 
considerando su relación con el Lenguaje de programación C

3. Emplear la IWU 2.0 durante la asignatura "Algorítmica y principios de programación" durante el ciclo 2014-3

4. Analizar el impacto de utilizar la IWU 2.0 durante el proceso de enseñanzaaprendizaje

A continuación se describe la metodología utilizada durante este proyecto de investigación.

\section{METODOLOGÍA}

Esta investigación se apoya en el Enfoque Cuantitativo con la finalidad de analizar el comportamiento de los estudiantes antes y después de utilizar la IWU 2.0 en la asignatura de Algorítmica y principios de programación. De acuerdo con Giroux y Tremblay (2004), este enfoque permite analizar los fenómenos por medio de la medición y análisis de datos con cifras.

El objetivo general de esta investigación es:

Diseñar e implementar los contenidos audiovisuales de la IWU 2.0 por medio de la accesibilidad para facilitar el proceso de enseñanza-aprendizaje sobre las Estructuras de Control

La muestra está conformada por 18 alumnos que estudian la Licenciatura ejecutiva en sistemas computacionales durante el ciclo escolar 2014-3 en una universidad ubicada al sur del Distrito Federal, México.

Según Elizondo (2002), el método experimental se basa en la producción deliberada de los fenómenos para analizar sus características. La variable independiente se refiere a la IWU 2.0 construida con base en la accesibilidad de los contenidos audiovisuales y la variable dependiente está conformada por el desarrollo del conocimiento relacionado con las Estructuras de control.

La investigación se desarrolla a partir de las siguientes hipótesis:

Hipótesis Nula (Ho): Los estudiantes que utilizan la IWU 2.0 no presentan un mejoramiento en el nivel de aprovechamiento (conocimiento) sobre las Estructuras de control y el Lenguaje de programación C.

Hipótesis Alternativa $(\mathrm{Ha})$ : Los estudiantes que utilizan la IWU 2.0 presentan un mejoramiento en el nivel de aprovechamiento (conocimiento) sobre las Estructuras de control y el Lenguaje de programación $\mathrm{C}$.

Para el caso de esta investigación, se aplicó el cuestionario diagnóstico sobre las Estructuras de control y el Lenguaje de programación C (preprueba) y otro cuestionario para analizar el comportamiento de los estudiantes después de emplear la IWU 2.0 durante el proceso de enseñanza-aprendizaje (postprueba). Es importante mencionar que la Prueba T permite a esta investigación analizar con un nivel de significancia del 0.05 y 0.1 los datos recopilados de los cuestionarios.

A continuación se describe los elementos vinculados con la accesibilidad de los contenidos audiovisuales empleados durante la construcción de la IWU 2.0. 


\subsection{Accesibilidad}

Hoy en día, las universidades en México buscan integrar nuevos métodos pedagógicos, tecnológicos y científicos que permitan planear e implementar innovadores escenarios virtuales que propicien el desarrollo eficiente de las competencias en los estudiantes. Por esta razón, los docentes analizan los avances que presentan las tecnologías de vanguardia durante el diseño y la construcción de los sistemas en la red. En particular, la accesibilidad de los contenidos audiovisuales ofrece una alternativa para crear sitios web que se adapten a las características de los usuarios.

Según Moreno, Martínez y Ruiz (2009) los criterios de la accesibilidad de los contenidos audiovisuales en la web (WCAG 2.0) presentan una relación estrecha con la usabilidad, la cual facilita el proceso de enseñanza- aprendizaje, la navegación en la red y proporciona información útil para el usuario por medio de los siguientes elementos:

1. Visibilidad del estado en el sistema

2. Flexibilidad y eficiencia de uso

3. Uso del lenguaje cotidiano

4. Facilidad para reconocer las funciones en el sistema

5. Control del sistema por el usuario

6. Prevención de errores

7. Empleo de estándares y consistencia en el diseño

8. Ofrecer ayuda al usuario durante la navegación

De acuerdo con Allsopp (2009), los estándares de la accesibilidad de los contenidos audiovisuales (WCAG 2.0) permiten adaptar de forma eficiente los avances de la tecnología en la web por medio de la aplicación de los 4 principios: perceptible, operable, comprensible y robusto.

Griol, Callejas y López (2013) explican los cuatro principios de la accesibilidad de los contenidos audiovisuales:

1. Perceptible: la información y los componentes de la interfaz debe ser presentada a los usuarios en diversas formas

2. Operable: los elementos que conforman la interfaz debe ser sencillos

3. Comprensible: los contenidos y las operaciones de la interfaz debe ser entendible para los usuarios

4. Robusto: El contenido y la interfaz deben ser empleadas por el mayor número de personas posibles

A continuación se describen los aspectos relacionados con la accesibilidad de los contenidos audiovisuales empleados durante la construcción e implementación de la IWU 2.0.

\section{DISCUSIóN}


La IWU 2.0 diseñada para esta investigación utiliza como uno de los aspectos innovadores a la accesibilidad de los contenidos audiovisuales. Como lo menciona Moreno (2008, p.93), "la Accesibilidad Web hace referencia a la capacidad de acceso a un sitio Web por todo tipo de usuarios, independientemente de sus discapacidades o su contexto de navegación, de modo que los usuarios serán capaces de percibir, entender, navegar e interactuar con dicho sitio".

En particular, la elaboración y la planeación de la información sobre las Estructuras de Control y el Lenguaje de programación $\mathrm{C}$ emplean las normas denominadas WCAG 2.0 creadas por el Consorcio Mundial de la Web (W3C) relacionadas con los aspectos: perceptible, operable, comprensible y robusta.

\subsection{Uso del principio perceptible en la IWU 2.0}

El primer principio sobre la accesibilidad en los contenidos audiovisuales establece que la información y los elementos de la interfaz deben presentarse en formas en las que los usuarios puedan distinguirlos. La Pauta 1.1 establece que se debe proporcionar las alternativas textuales. El diseño de la IWU 2.0 permite a los estudiantes controlar los subtítulos durante la ejecución del material interactivo sobre las Estructuras de control por medio del ratón y teclado. La Figura 2 muestra el diagrama empleado para la construcción del IWU 2.0.

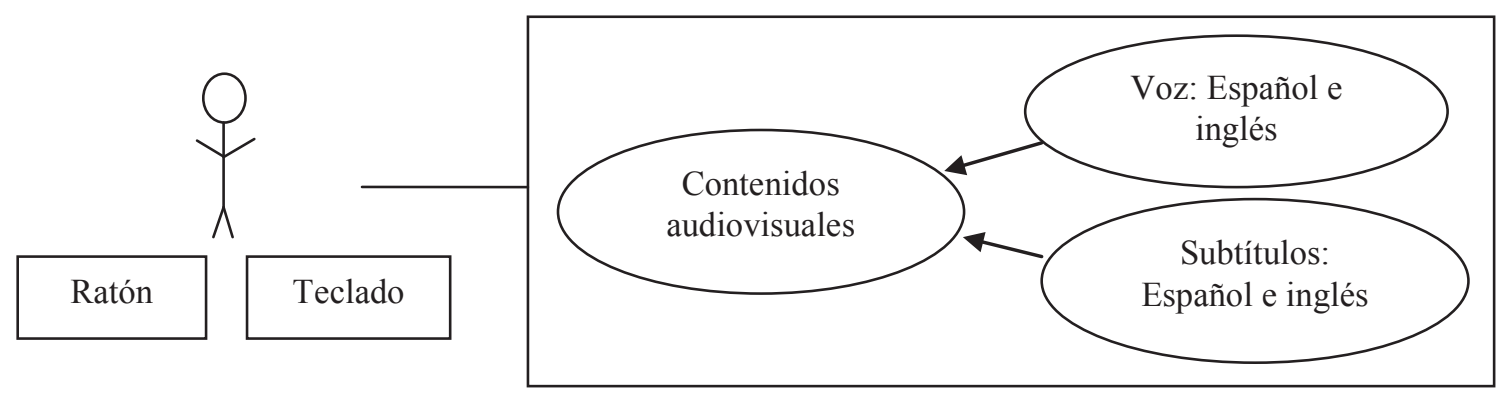

Figura 2: Empleo de la Pauta 1.1 sobre la accesibilidad en la IWU 2.0

Fuente: Diseño propio 2014

La Pauta 1.2 sobre la accesibilidad establece que se debe proporcionar alternativas para el contenido audiovisual en el tiempo. En particular, la IWU 2.0 permite al usuario configurar la interfaz Web a través de la voz y los subtítulos durante su ejecución. (Ver Figura 3). 


\section{Secuencia}

\section{Secuencia}

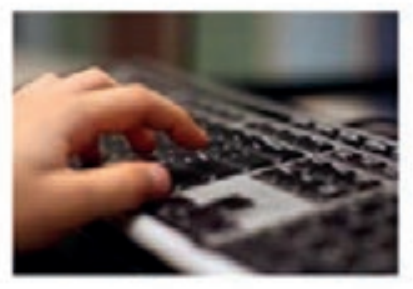

La estructura de control (Secuencia)

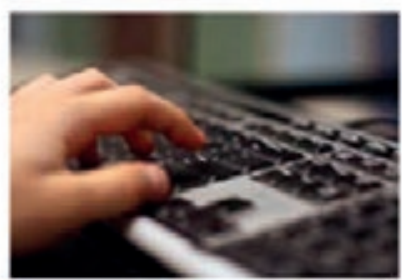

Instrucción 1

Instrucción 2

Instrucción 3

Control structure(sequence)

Figura 3: Empleo de la Pauta 1.2 sobre la accesibilidad en la IWU 2.0

Fuente: Diseño propio 2014

La Pauta 1.3 establece que el contenido audiovisual debe ser presentado en diversas formas sin perder información. Por lo tanto, el usuario puede configurar la plataforma educativa para observar únicamente la trascripción de la información o escuchar el diálogo del video manteniendo la misma estructura (Ver la Figura 4).
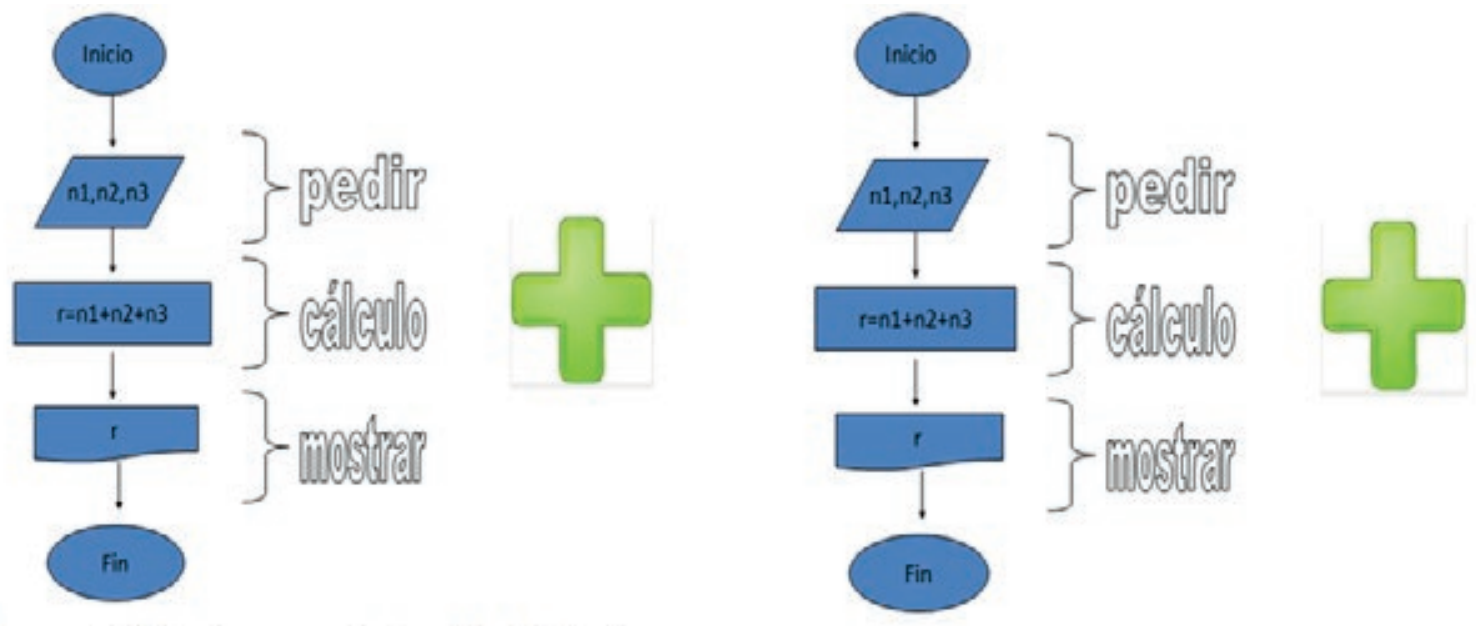

El diagrama de flujo es la representación gráfica del algoritmo.

En este caso se muestra la suma de tres números.

Figura 4: Empleo de la Pauta 1.3 sobre la accesibilidad en la IWU 2.0

Fuente: Diseño propio 2014

Finalmente, la Pauta 1.4 establece que se debe facilitar a los usuarios ver y escuchar los datos. En este sentido, la IWU 2.0 presenta la sección de ayuda y permite al usuario configurar los temas sobre Estructuras de control por medio del ratón o del teclado.

La Figura 5 muestra la sección de ayuda donde se describen las acciones que se puede realizar en la IWU 2.0 a través del teclado (acceso rápido) o ratón. 


\section{CONFIGURACIÓN}

$\begin{array}{ll}\text { Voz : } & \begin{array}{l}\text { Español } \\ \text { Hombre }\end{array} \\ \text { Subtitulos: } & \text { Español } \\ \text { Control : } & \text { Voz } \\ \text { Acceso: } & \text { Flecha }\end{array}$

Figura 5: Empleo de la Pauta 1.4 sobre la accesibilidad en la IWU 2.0

Fuente: Diseño propio 2014

\subsection{Uso del principio operable en la IWU 2.0}

Según Moreno (2008), el segundo principio sobre la accesibilidad establece que los componentes de la interfaz y la navegación deber sencillos, esto es, la Pauta 2.1 menciona que el teclado es empleado para la interacción entre el usuario y la información. En particular, el alumno universitario puede cambiar la configuración y controlar el producto multimedia web de la IWU 2.0 a través del teclado. La Figura 6 describe el acceso rápido empleado en esta investigación.

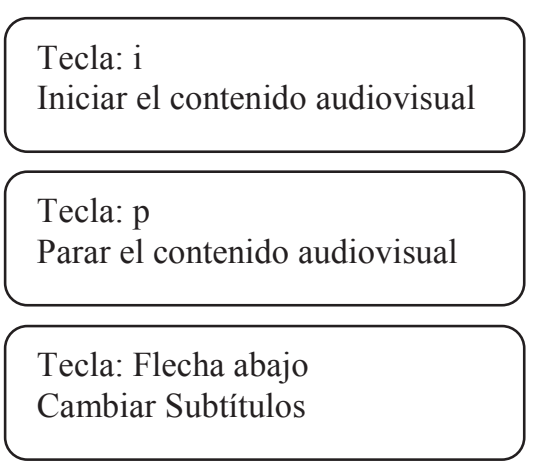

\section{Tecla: Flecha derecha \\ Avanzar el contenido audiovisual}

Tecla: Flecha izquierda

Regresar el contenido

Tecla: Flecha arriba

Cambiar voz
Tecla: a

Aumentar sonido

Tecla: d

Disminuir sonido

Figura 6: Empleo de la Pauta 2.1 sobre la accesibilidad en la IWU 2.0

Fuente: Diseño propio 2014

La Pauta 2.2 sobre el contenido operable establece que se debe proporcionar a los usuarios suficiente tiempo para leer y usar el contenido. En este sentido, el diseño del producto interactivo sincroniza la voz con el texto para transmitir eficientemente el mensaje educativo (Ver Figura 7).

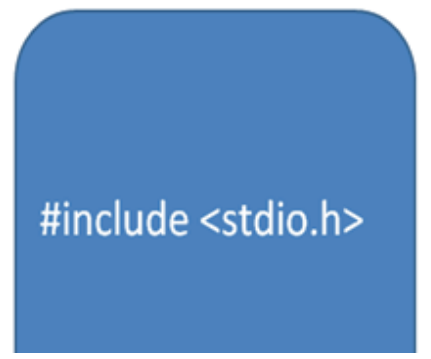

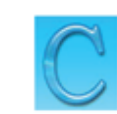

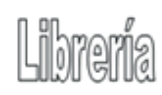

int num1;

int num2;
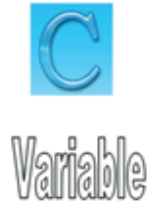
Figura 7: Empleo de la Pauta 2.2 sobre la accesibilidad en la IWU 2.0

Fuente: Diseño propio 2014

Por último, la Pauta 2.3 establece que no se debe diseñar el contenido en formas que se conocen que pueden provocar ataques epilépticos y la Pauta 2.4 menciona que se debe proporcionar distintas formas de ayudar a los usuarios a navegar y determinar donde están. La Figura 8 muestra la IWU 2.0.

\section{Algorítmica y principios de programación}

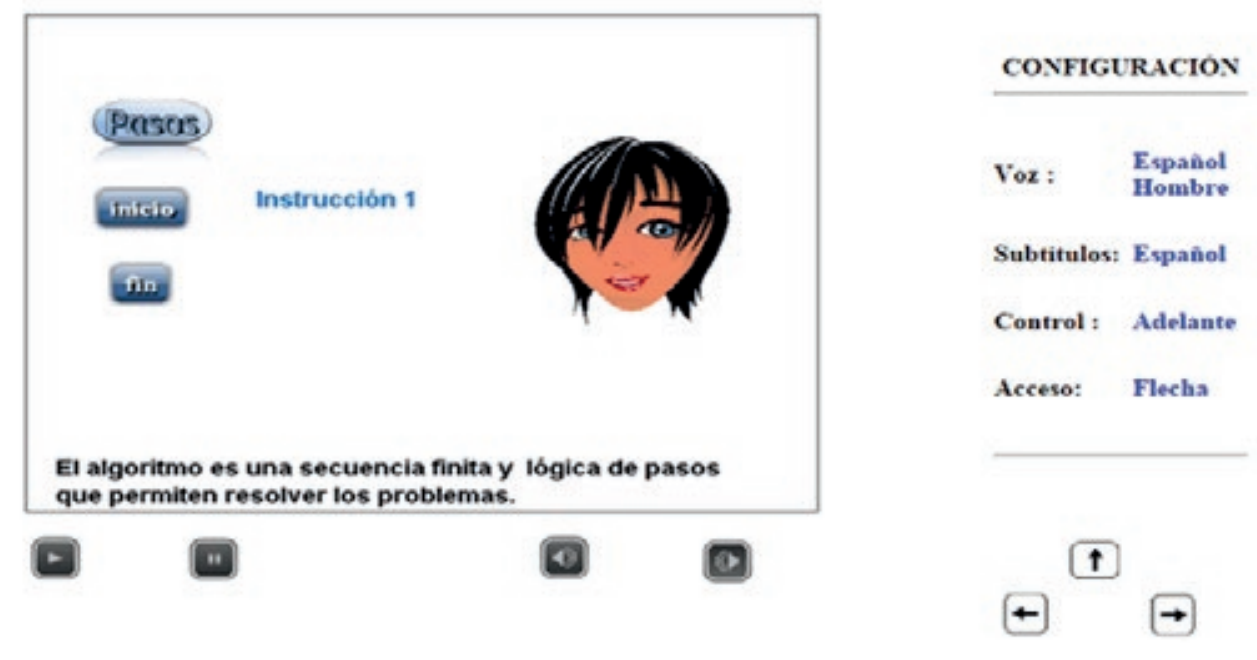

Figura 8: Empleo de la Pauta 2.3 y 2.4 sobre la accesibilidad en la IWU 2.0 Fuente: Diseño propio 2014

\subsection{Uso del principio comprensible en la IWU 2.0}

El tercer principio sobre la accesibilidad de los contenidos audiovisuales se refiere a que la información y el manejo de la interfaz deben ser claros. En particular, la Pauta 3.1 establece que el contenido textual debe ser legible y comprensible. Por esta razón, la planeación e implementación de la IWU 2.0 se apoya en la selección adecuada de la identidad gráfica con la finalidad de lograr el aprendizaje significativo en los estudiantes (Ver Figura 9). 


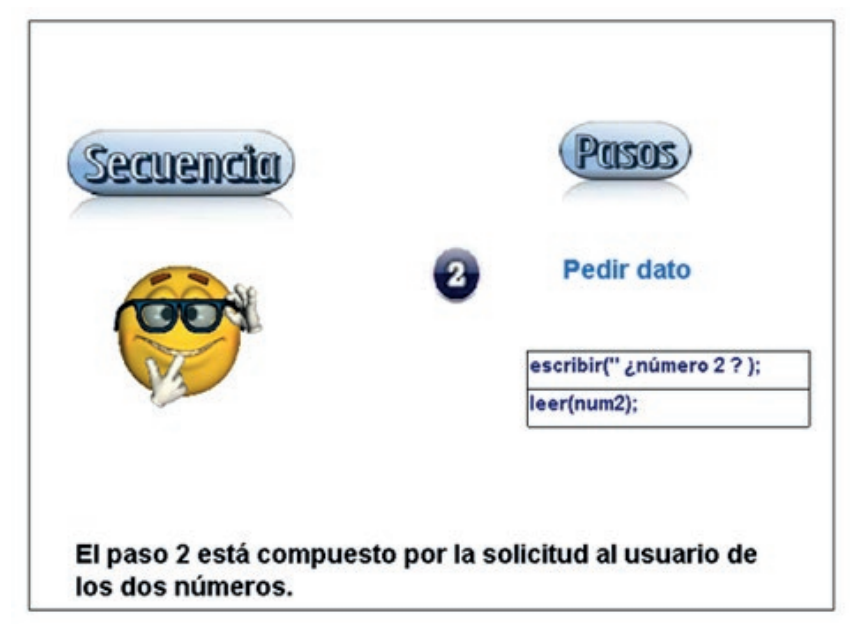

Figura 9: Empleo de la Pauta 3.1 sobre la accesibilidad en la IWU 2.0 Fuente: Diseño propio 2014

La selección adecuada del color, el tamaño y estilo de la fuente y las imágenes permiten crear escenarios virtuales adecuados para la transmisión del mensaje. De acuerdo con Alberich (2007) es fundamental el establecimiento homogéneo de un código gráfico (elementos gráficos e imágenes), código tipográfico (familia tipográficas, jerarquías, márgenes) y código cromático (paletas de colores).

Durante el diseño de la IWU 2.0 se considera la familia tipográfica, las jerarquías y los márgenes para la construcción de la interfaz Web.

Además Gallego (1999) explica que la regla del $20 \%$ consiste en categorizar el uso de los tamaños y grosores. Sklar (2009) explica que para crear un diseño efectivo se necesitan seleccionar pocos estilos y tamaños de letra, emplear las fuentes disponibles y diseñar empleando la legibilidad. Por consiguiente, las letras utilizadas en el diseño de los contenidos IWU 2.0 pertenecen a la familia de fuentes "Sansserif".

Otro de los factores que se debe considerar durante el diseño los contenidos audiovisuales compresibles es el código cromático. De acuerdo con Sharpless (2006), el color es uno de los elementos visuales más importantes para el diseño porque éste guía al usuario hacia un lugar en específico dentro la interfaz y permite establecer diferencia entre los elementos (Ver Tabla 1).

Tabla 1

Psicología del Color

\begin{tabular}{cccccc}
\hline Rojo & Azul & Verde & Gris & Blanco & Negro \\
\hline poder & paz & naturaleza & inteligencia & puro & riqueza \\
energía & tranquilidad & salud & modestia & precisión & formalidad \\
pasión & calma & buena suerte & madurez & inocencia & enojo \\
amor & armonía & ambiente & futuro & muerte & poder \\
\hline
\end{tabular}

Tomado de Sharpless, 2006. 
De acuerdo con Fernández (1998), el empleo de diversos colores confunde al usuario el cual no sabrá el lugar que debe mirar con atención. Es decir, es conveniente definir tres o cuatro colores básicos y usar sus combinaciones de tono. Por consiguiente, la planeación de la IWU 2.0 emplea tres colores básicos: blanco, negro y azul La Figura 10 muestra el uso del código gráfico, tipográfico y cromático en la IWU 2.0.

\section{Algorítmica y principios de programación}

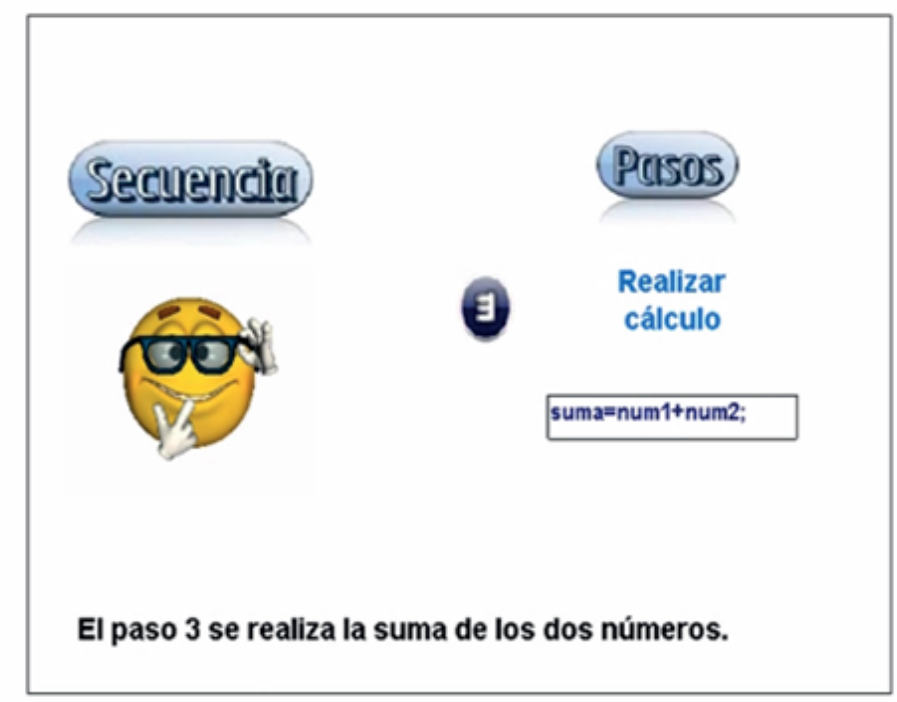

CONFIGURACIÓN

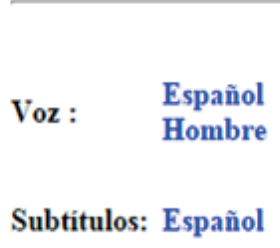

Control :

Acceso:

Figura 10: Empleo del código gráfico, tipográfico y cromático en la IWU 2.0

Fuente: Diseño propio 2014

Del mismo modo, la Pauta 3.2 sobre los contenidos audiovisuales comprensibles establece que las páginas Web se deben controlar de manera predecible. En consecuencia, la construcción del producto interactivo multimedia utiliza imágenes fáciles de reconocer como las flechas o los iconos de play y stop.

\subsection{Uso del principio robusto en la IWU 2.0}

Este principio sobre la accesibilidad en los contenidos audiovisuales se refiere a que la información pueda ser interpretada por una amplia variedad de individuos. En particular, la Pauta 4.1 establece maximizar la compatibilidad con los agentes de usuarios actuales y futuros, incluyendo los productos de apoyo. Por consiguiente, el diseño del IWU 2.0 emplea como elemento novedoso al lenguaje HTML 5 para lograr transmitir el conocimiento al mayor número de usuarios.

Como lo menciona Meyer (2010), este lenguaje presenta nuevas características que facilitan la forma de transmitir la información en Internet. Por ejemplo, la etiqueta <video> transmite los contenidos audiovisuales en diversos navegadores como Internet Explorer, Firefox, Opera, Chrome y Safari por medio de los formatos ogg, 
mp4 y webm. También admite el acceso de la información en la red a través de distintos sistemas operativos como Linux, Mac y Windows.

JavaScript permite controlar las actividades del sitio Web a través de la programación. En particular, este programa permite crear botones para administrar el video, las animaciones y el sonido de acuerdo a las necesidades de los estudiantes En consecuencia, la organización del IWU 2.0 se apoya en los avances tecnológicos relacionados con HTML 5 y JavaScript para crear un escenario que propicie el aprendizaje significativo en los estudiantes.

\subsection{Resultados}

Por medio de la hoja de cálculo se realiza la Prueba T con el nivel de significancia de 0.05 y 0.1 . La Tabla 2 muestra que la media de la preprueba es de 5.38 y la media de la postprueba de 8.22 .

\section{Tabla 2}

Prueba T con el nivel de significancia de 0.05

\begin{tabular}{lrr}
\hline & Preprueba & Postprueba \\
\hline Media & 5.38888889 & 8.22222222 \\
Varianza & 4.36928105 & 1.12418301 \\
Observaciones & 18 & 18 \\
Varianza agrupada & 2.74673203 & \\
Diferencia hipotética de las medias & 0 & \\
Grados de libertad & 34 & \\
Estadístico t & 5.12874114 & \\
P(T<=t) dos colas & $1.1694 \mathrm{E}-05$ & \\
Valor crítico de t (dos colas) & 2.0322445 & \\
\hline
\end{tabular}

Elaboración propia, 2014.

Como se muestra en la Tabla 2, la hipótesis nula es rechazada debido a que el valor crítico de t para dos colas (2.03) es mayor que el valor estadístico. Por esta razón, se puede concluir que la IWU 2.0 mejora el nivel de aprovechamiento de los estudiantes que cursan los contenidos relacionados con las Estructuras de control y el Lenguaje de programación $\mathrm{C}$.

La Tabla 3 muestra los resultados de la Prueba T con el nivel de significancia de 0.1 donde nuevamente es rechazada la hipótesis nula y es aceptada la hipótesis alternativa.

Tabla 3

Prueba T con el nivel de significancia de 0.1

\begin{tabular}{lcc}
\hline & Preprueba & Postprueba \\
\hline Media & 5.38888889 & 8.22222222
\end{tabular}


Varianza

Observaciones

Varianza agrupada

Diferencia hipotética de las medias

Grados de libertad

Estadístico $\mathrm{t}$

$\mathrm{P}(\mathrm{T}<=\mathrm{t})$ dos colas

Valor crítico de t (dos colas)

Elaboración propia, 2014.
$4.36928105 \quad 1.12418301$

18

18

2.74673203

0

34

$-.12874114$

1.1694E-05

1.6909242

En resumen, la Prueba $T$ con los niveles de significancia de 0.1 y 0.05 permite afirmar que el uso de la IWU 2.0 mejora el nivel de aprovechamiento de los estudiantes respecto a los contenidos de las Estructuras de control y el Lenguaje de programación C.

\section{CONCLUSIONES}

Los docentes junto con las instituciones educativas deben de proponer nuevas alternativas tecnológicas que faciliten el proceso de enseñanza-aprendizaje con la finalidad de que los estudiantes desarrollen de forma eficiente sus competencias. En particular, el IWU 2.0 es una interfaz web educativa que le permite al usuario controlar la forma de presentar los contenidos audiovisuales sobre las Estructuras de control y el Lenguaje de programación C por medio de la selección de la voz y las alternativas textuales.

Es importante que los estudiantes cuenten con el apoyo de diversos recursos didácticos vinculados con la tecnología de vanguardia que les permitan entender y aplicar los conocimientos de las asignaturas en los contextos apegados a la realidad. En el caso de esta investigación, la IWU 2.0 permitió a los alumnos que cursan la materia Algorítmica y principios de programación mejorar su aprovechamiento (conocimiento) debido a la personalización de la información de acuerdo a sus características.

La accesibilidad de los contenidos audiovisuales es un elemento que debe ser considerando durante el diseño y la implementación de los sistemas debido a que facilita la transmisión de los datos perceptibles, operables, comprensibles y robustos. Por ejemplo, el uso de alternativas textuales apoya a los estudiantes visuales a comprender los contenidos, el teclado permite a los usuarios controlar la interfaz web de forma rápida, sencilla e intuitiva y la estética facilita la difusión del mensaje educativo.

Asimismo, se recomienda el diseño de la interfaz web considerando al lenguaje de HTML5 debido a que permite ofrecer al usuario flexibilidad en los navegadores y sistemas operativos.

Finalmente, los docentes deben de estar en constante capacitación con el propósito de mejorar sus conocimientos y habilidades vinculadas con el uso de la tecnología en la educación para lograr la creación de espacios innovadores virtuales que mejoren el 
proceso de enseñanza-aprendizaje. Por ejemplo, IWU 2.0 brinda a los estudiantes la posibilidad de recibir la información relacionada con las Estructuras de control y el Lenguaje de programación C considerando sus características.

\section{REFERENCIAS}

\section{LIBROS, CAPÍTULOS DE LIBRO O ENTRADA DE UN LIBRO DE CONSULTA, INFORMES TÉCNICOS, TESIS}

Alberich Pascual, J. (2007). Grafismo multimedia. Comunicación diseño, estética. Barcelona, España: UOC

Allsopp, J. (2009). Developing with web standards. Estados Unidos: New Riders

Elizondo López, A. (2002). Metodología de la investigación. Distrito Federal, México: Thomson

Fernández Coca, A. (1998). Producción y diseño gráfico para la World Wide Web. Barcelona, España: Paidos.

Gallego Gil, D. J. (1999). Multimedia en la Web. Madrid, España: Dykinson

Giroux, S. y Tremblay G. (2004). Metodología de las Ciencias Humanas. Distrito Federal, México: Fondo de Cultura Económica

Griol Barres, D., Callejas Carrión, Z. y López Cózar , Ramón (2013). Technologies for inclusive education: Beyond traditional integration approaches. Estados Unidos: IGI Global

Meyer, J. (2010). The essential guide to HTML 5. Using games to learn HTML5 and JavaScript. Nueva York,Estados Unidos: Friendof

Moreno, L. (2008). Accesibilidad a los contenidos audiovisuales en la Web. Madrid, España: Imagen Gráfica

Sharpless Smith, S. (2006).Web-based instruction. A guide for libraries. Estados unidos: McNaughton \& Gunn

Sklar, J. (2009). Principles of Web Design. Canadá: Cengage Learning

PUBLICACIONES PERIÓDICAS, REVISTAS, WEBES Y SIMILARES: 
Moreno, L., Martínez, P., Ruiz Mezcua, B. (2009). A bridge to web accessibility from the usability heuristics. En A. Holzinger y K. Miesenberger (Eds.), HCI and usability for e-inclusion, pp. 290-300: Springer

Shirogane, J., Mori, T.,Iwata, H. y Fukazawa, Y. (2008). Accesibility : Evaluation for GUI software using source programs. En M. Virvou y T. Nakamura (Eds), Knowledge-Based Software Engineering, pp. 135-144, IOS Press

\section{AUTOR:}

\section{Ricardo Adán Salas Rueda}

Doctor en Diseño de Nuevas Tecnologías, egresado de la Universidad Autónoma Metropolitana (UAM) en el año 2014. Durante 13 años ha impartido diversos cursos relacionados con la tecnología, la administración, las matemáticas en el nivel de licenciatura y posgrado. Estudió la Maestría en Administración de Empresas y la Ingeniería en Sistemas Electrónicos. 